\title{
The design characteristic of VIP terminal of airport
}

\author{
Liu Huihui ${ }^{1}$ \\ ${ }^{1}$ Airport Planning and Design Institute, China Aviation International Construction and Investment Co., Ltd., Beijing, \\ 100120, China
}

\begin{abstract}
This article discusses the key points of the VIP terminal design, and discusses in detail the difference between VIP terminal and the large-scale terminal from the aspects of passengers, scale, service, and plane functions.
\end{abstract}

\section{The development of VIP terminal}

With the continuous improvement of the aviation market, more and more people choose flying as the way to their long-distance travel. The difference between high-end passengers and regular passengers on flight demand is increasingly prominent, so it is necessary to customize suitable waiting space and environment. This small-scale terminal providing high-end services is called VIP terminal. The service objects of VIP terminal include high-end business personnel, government personnel, as well as all kinds of people who need charter flights. VIP terminal fully meets the high-end needs of passengers for privacy, quickness and comfort, and improves the service quality for passengers.

With the advantages of efficient and convenient travel, VIP airplane is favoured by more and more people in China. The fleet size of China's VIP airplane market ranks first in the Asia Pacific market, and is still growing at a rate of $10 \%$ every year.

Since the outbreak of the new crown epidemic this year, VIP airplane has become the first choice of many people for long-distance travel due to its characteristics of small passenger capacity, private space and exclusive access. The demand for VIP airplane has increased significantly. It can be predicted that the VIP airplane market will continue to increase in the future, and the demand for VIP terminal will also increase year by year.

\section{The design features of VIP terminal}

\subsection{The features of the plan function}

Lager terminal often need to consider the complex transportation system, including expressway, urban rail, etc., and become the comprehensive transportation hub of the city. ${ }^{[1]}$.

There are few people and passenger vehicles in the VIP terminal, so there is no need for complex transfer system. The ground road is often used in front of the building to solve the most basic air land transfer problem.

With the increase of annual passenger volume, the functional process of large-scale terminal is more complex. The plane functional space includes departure and arrival hall, luggage check-in hall, security check-in, near/far departure hall, commerce, exhibition and office logistics. ${ }^{[2]}$.

The service object of the VIP terminal is a special VIP group with few passengers. Therefore, it is necessary to set up an independent boarding process, which is generally simpler and more efficient than that of a large terminal. There is a separate boarding process for accompanying personnel and luggage. The waiting part includes VIP space with diversified supporting services and separate service personnel.

\subsection{Pay attention to service}

More and more terminal begin to pay attention to the feelings of passengers, introducing greening, commerce, catering, entertainment, exhibition, to provide passengers with the dual enjoyment of vision and experience. Green, environmental protection and humanistic experience have become the new design concept of the terminal. The whole terminal becomes a huge exhibition hall and the centre of shopping and entertainment. (Figure 1$)^{[3]}$. 


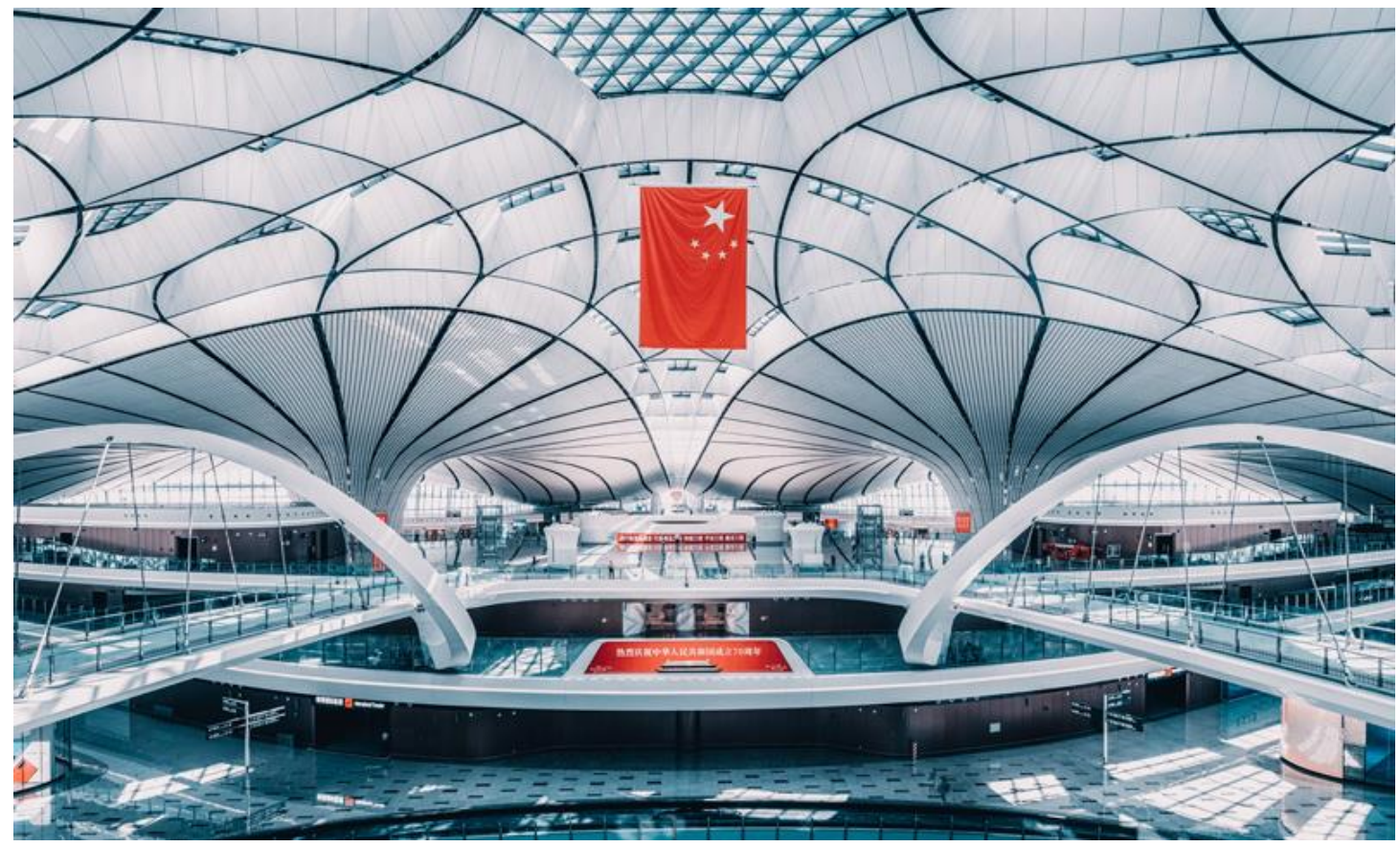

Figure 1 Daxing airport terminal, Beijng

Due to the particularity of the service objects, there are more high-end passengers and government personnel in the VIP terminal, whose demands are obviously different from those of regular passengers. In order to meet the needs of those high-end passengers, VIP terminal has introduced the multi-function meeting hall, VIP meeting room, the hotel and even more functions for the government. (Figure 2$)^{[4]}$.

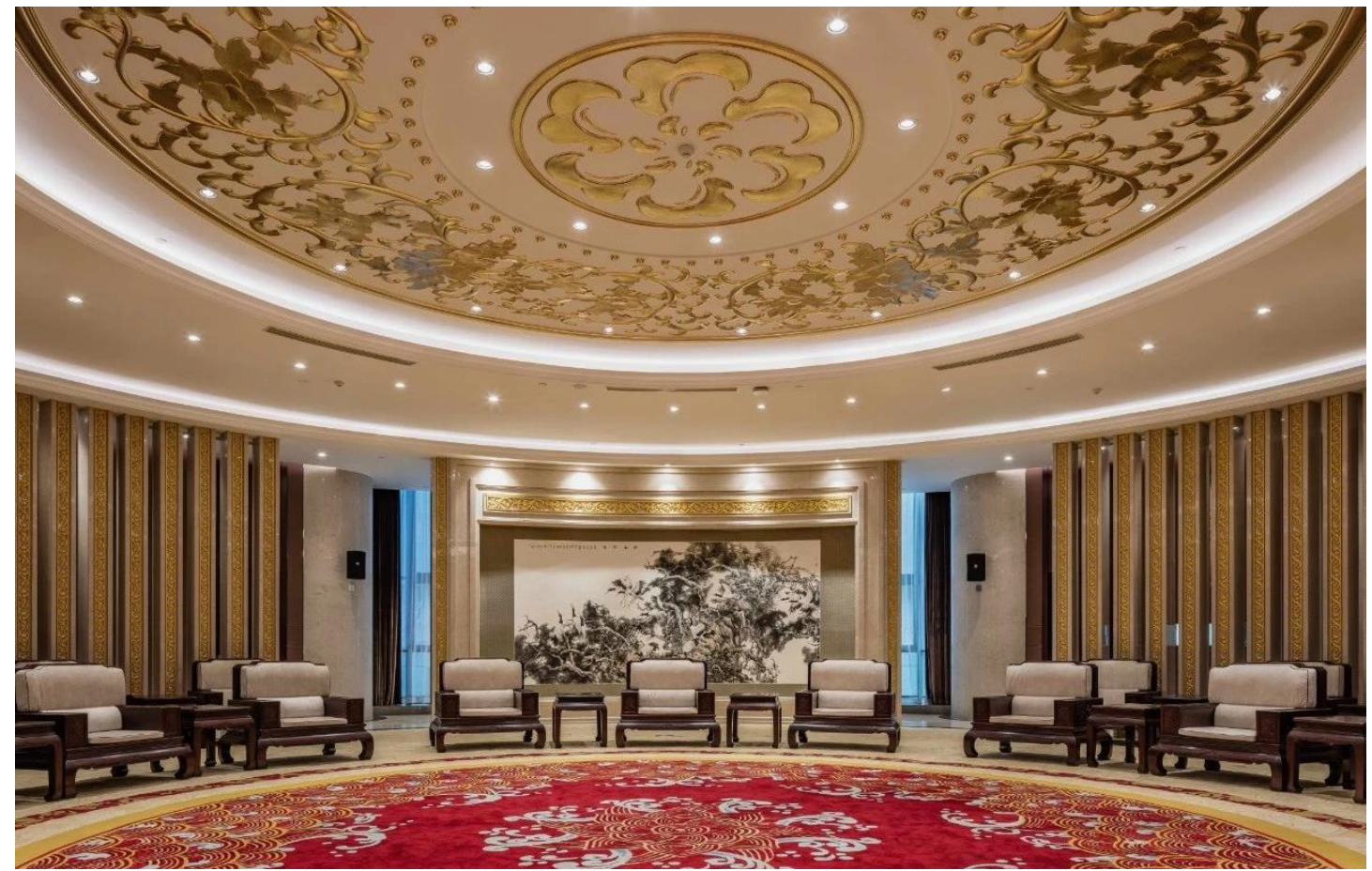

Figure 2 the multi-function meeting hall in VIP terminal, Kunming

buildings adopt modern architectural language, which is

\subsection{Diversified language of building facade}

Due to its huge volume, most of the large terminal composed of steel structure and glass curtain wall. (Figure 3$)^{[5]}$. 

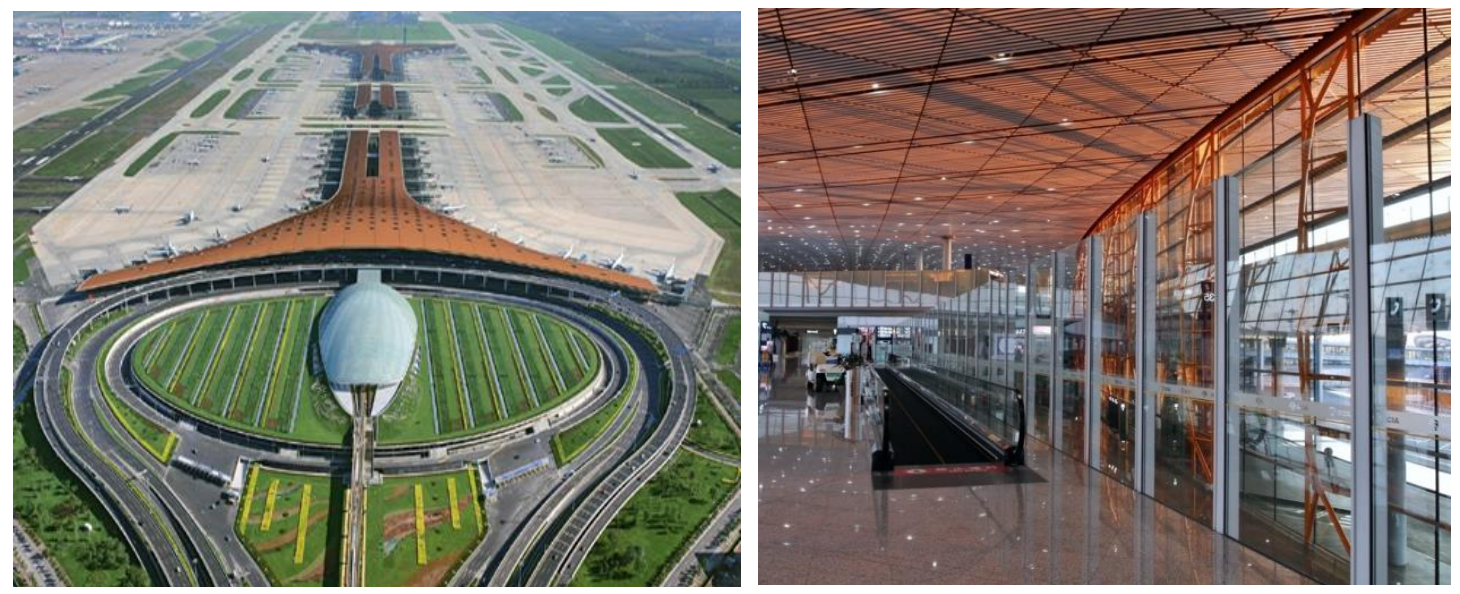

Figure 3 terminal 3 of the capital airport, Beijng

The VIP terminal is generally small in size, flexible in architectural language, and highly cultural and recognizable in respect of the city's geography and culture. The architectural style of the VIP terminal of Beijing Capital Airport is "Chinese neoclassical", reflecting the characteristics of China's times. (Figure 4 )

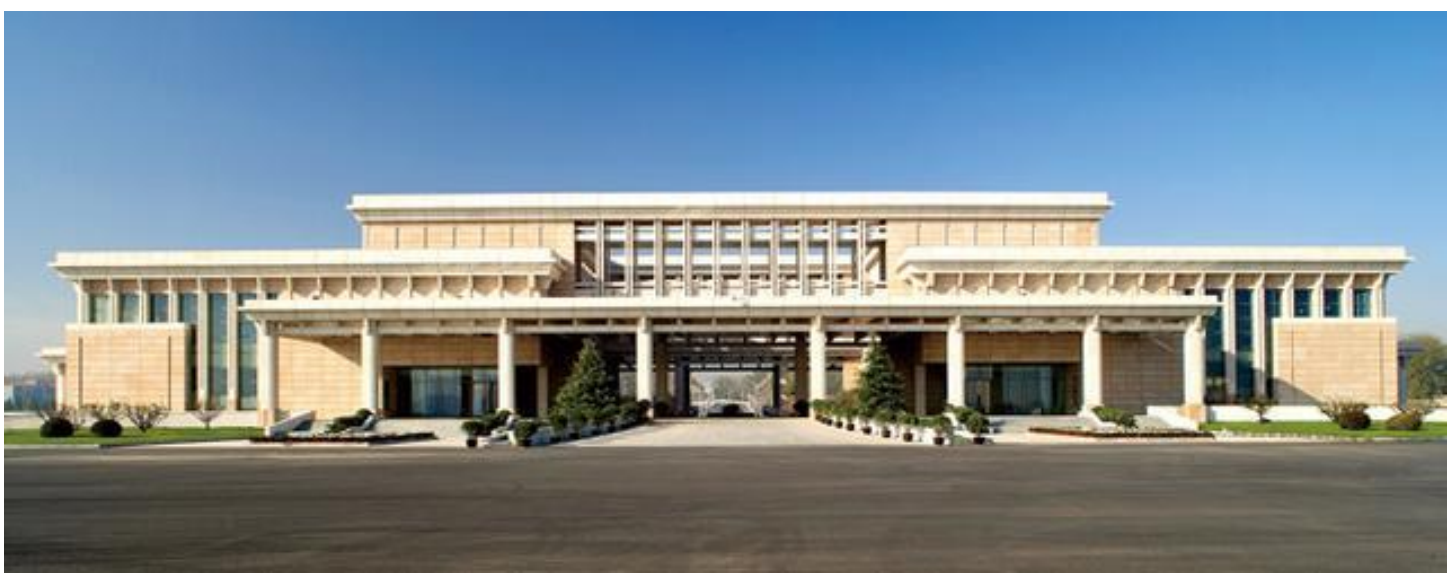

Figure 4 VIP terminal of the capital airport, Beijng

\section{In conclusion}

The development of China's VIP airplane industry is still in its infancy and has great development potential. At present, the development speed of VIP airplane industry has been limited by the lack of infrastructure. As the necessary supporting service facilities for VIP airplane, the characteristics of VIP terminal are worth thinking about, which can provide reference for future design.

\section{References}

1. Wang,X.Q. (2020) From BCIA to BDIA, One Decade of the Development of Airport Terminals . J. World Architecture, 06:pp, 47.

2. Qiu,X.Y.,Tan,B.,Pan,L. (2020) Exploring the Prospective Trends of Large-Scale Airport Terminals. J. World Architecture, 06:pp, 35.

3. Liu,Y.,Zhong,L.,Chen,R.F. (2019) Design research on spatial comfort and green energy saving of airport terminals. J. Architectural journal, 09:pp, 22-23.
4. Guo,J.X.,Yang.X (2020) Rapidly Changing Design of Modern Terminal Building. J. World Architecture, 06:pp, 21. 